EXTENDED REPORT

\title{
Application of femtosecond ultrashort pulse laser to photodynamic therapy mediated by indocyanine green
}

\author{
M Sawa, K Awazu, T Takahashi, H Sakaguchi, H Horiike, M Ohii, Y Tano
}

Br J Ophthalmol 2004;88:826-831. doi: 10.1136/bjo.2003.031047

See end of article for authors' affiliations

......................

Correspondence to: Dr M Sawa Department of Ophthalmology and Visual Science, Osaka University Graduate School of Medicine, Room E7, 2-2 Yamadaoka, Suita, Osaka, Japan 565-0871: sawamiki@ophthal.med. osaka-u.ac.jp

Accepted

3 November 2003
Backgrounds/aims: To evaluate treatment with high peak power pulse energy by femtosecond ultrashort pulse laser (titanium sapphire laser) delivered at an $800 \mathrm{~nm}$ wavelength for corneal neovascularisation using photodynamic therapy (PDT) mediated by indocyanine green (ICG).

Methods: Using a gelatin solid as an in vitro corneal model, the safety of laser power was studied to determine if it degenerated gelatin with or without ICG. The authors then induced corneal neovascularisation in rabbit eyes by an intracorneal suturing technique. Fluorescein angiography was used to evaluate occlusion before PDT and 0, 1, 3, and 10 days after PDT. The authors performed light microscopy with haematoxylin eosin staining and transmission electron microscopy to determine thrombosis formation in the neovascular regions.

Results: The threshold of peak laser power density ranged from 39 to $53 \mathrm{~W} / \mathrm{cm}^{2}$. Laser irradiation was started 30 seconds after a $10 \mathrm{mg} / \mathrm{kg}$ ICG injection, and all irradiated segments were occluded at $0,1,3$, and 10 days at $3.8 \mathrm{~J} / \mathrm{cm}^{2}$. Light and electron microscopy documented thrombosis formation in the neovascular region.

Conclusion: Femtosecond pulse laser enhanced by ICG can be used for PDT. Because of effective closure of corneal neovascularisation at a low energy level, the high peak power pulse energy of the femtosecond pulse laser might be more efficacious than continuous wave laser for use with PDT.
V arious types of lasers have been used as treatment modalities in ophthalmology, but clinicians primarily use continuous krypton or diode wave lasers. The femtosecond pulse laser is advantageous in that it produces high peak power pulse energy (individual pulse energy divided by the pulse duration), and it provides a new type of three dimensional imaging using two photon absorption, which produces a confocal microscopic image such as that in optical coherence tomography. Recently, a femtosecond pulse laser was used to show photodisruption with less collateral damage compared with nanosecond pulse lasers. ${ }^{1}$

The titanium sapphire laser, a type of femtosecond pulse laser, has a wavelength in the near infrared level (range 780$1000 \mathrm{~nm}$ ) and can be frequency doubled or frequency tripled to access wavelengths in the visible and ultraviolet spectral regions. ${ }^{2}$ In refractive surgery, the femtosecond pulse laser is beneficial because it causes less bubble formation generated from shock waves than nanosecond or picosecond pulse lasers. ${ }^{3}$ The femtosecond infrared titanium sapphire laser has been applied to enhance an in vivo gene delivery procedure. ${ }^{4} 5$

Photodynamic therapy (PDT), an established procedure that induces thrombus formation and occludes microvasculature with minimal damage to the surrounding normal tissue, is also used in treating ocular neovascularisation. ${ }^{6} \mathrm{~A}$ second generation photosensitiser, liposomal benzoporphyrin derivative, was investigated ${ }^{7}$ and clinically applied to age related macular degeneration. ${ }^{8}$ Although liposomal benzoporphyrin derivative has a shorter half life than the first generation photosensitisers-haematoporphyrin derivatives-skin photosensitivity might occur. PDT mediated by liposomal benzophorphyrin derivative does not produce the best improvement in visual acuity and requires frequent retreatment. The treatment cost is a problem because the photosensitiser is expensive.

Indocyanine green (ICG), which has an excellent safety record with few adverse effects, ${ }^{9}$ has contributed to the diagnosis of diseases such as choroidal neovascularisation. ${ }^{10}$
Recently, ICG was applied as a photosensitiser for PDT in vitro $^{11}{ }^{12}$ and in vivo. ${ }^{13}{ }^{14}$ ICG could be a candidate for use as a photosensitiser with minimal adverse effects. ICG has an absorption peak at $805 \mathrm{~nm}$, which is similar to the wavelength of the titanium sapphire laser at the near infrared level.

We combined high peak power pulse energy, which causes less collateral damage by the titanium sapphire laser, with PDT mediated by ICG. To evaluate this combination, corneal neovascularisation was used as a neovascularisation model.

\section{MATERIALS AND METHODS}

\section{Femtosecond pulse laser}

A Spectra Physics Hurricane laser (Mountain View, CA, USA), consisting of the Mai-Tai laser, Evolution laser, and Spitfire regenerative amplifier, generated 150 femtoseconds at $800 \mathrm{~nm}$ and an average power of $730 \mathrm{~mW}$. The laser operated at a $1 \mathrm{kHz}$ pulse repetition rate. The energy stability was typically within $2 \%$, thereby providing a high quality beam intensity profile for uniform interaction on the target. This femtosecond laser pulse duration is shorter than the characteristic energy relaxation times-that is, the electron to ion energy transfer and the heat conduction time.

\section{Absorption properties of ICG solutions}

The ultraviolet (UV)/(VIS) absorption spectra of the ICG solution (Diagnogreen, Dai ichi Pharmaceutical Co Ltd, Tokyo, Japan) were measured by UV/VIS spectrometer (U3500, Hitachi, Tokyo, Japan). An ICG solution with a water solvent has absorption peaks at $710 \mathrm{~nm}$ and $780 \mathrm{~nm}$ in the visible light range. The absorption peaks shift to $805 \mathrm{~nm}$ in plasma protein.

Indocyanine green containing less than $5 \%$ iodine was dissolved in distilled water at concentrations of $0.1,0.5$, and $1.0 \mathrm{mg} / \mathrm{dl}$, and $0.5 \mathrm{mg} / \mathrm{dl}$ of albumin was added. Absorption

Abbreviations: ICG, indocyanine green; PDT, photodynamic therapy 
was measured by spectrophotometry (Diaphot 300, Nikon, Tokyo, Japan). Absorbance was measured at the $805 \mathrm{~nm}$ wavelength with each concentration of the ICG solution.

\section{Threshold of laser power with gelatin with and without ICG}

We investigated the irradiation effects of the femtosecond laser on gelatin containing ICG. The laser was focused on the inside of the gelatin target using a $250 \mathrm{~mm}$ focal length refracting mirror to a focal spot of about $0.2 \mu \mathrm{m}$ in full width at half maximum (focal spot size area, $0.047 \mathrm{~cm}^{2}$ ), providing a maximum laser intensity of about $10^{14} \mathrm{~W} / \mathrm{cm}^{2}$ with 150 femtosecond pulses (maximum laser fluency of $1 \mathrm{~J} / \mathrm{cm}^{2}$ ). The laser intensity was varied by inserting neutral density filters into the beam. The laser beam focused in the gelatin and formed the denatured area.

Gelatin (Sigma Chemical, St Louis, MO, USA) (20 mg) was dissolved in $180 \mathrm{ml}$ distilled water. Gelatin was coagulated in a $1 \mathrm{~cm}$ cell. The mean power density was measured to degenerate the gelatin. Using gelatin mixed with $1.5 \mathrm{mg} / \mathrm{dl}$ of ICG, the mean power density also was measured.

\section{Corneal neovascularisation}

This study was conducted in accordance with the Association for Research in Vision and Ophthalmology Statement for the Use of Animals in Research.

To evaluate the effect of PDT using a femtosecond pulse laser mediated by ICG, a corneal neovascularisation model in rabbit eyes was used, which is an established model using the technique of intracorneal sutures. ${ }^{15}$ Thirty one albino rabbits weighing $2-2.5 \mathrm{~kg}$ were used. Corneal neovascularisation was induced in one eye of each animal using an intracorneal suturing technique as previously described. ${ }^{716}$ Briefly, the rabbits were anaesthetised with intramuscular injections of ketamine hydrochloride $(35 \mathrm{mg} / \mathrm{kg})$ and xylazine hydrochloride $(5 \mathrm{mg} / \mathrm{kg}$ ). Silk sutures (size 7-0) were placed to midstromal depth radially at the 11,12 , and 1 o'clock positions on the cornea. The sutures started from the limbus and advanced toward the centre of the cornea. None of the knots was buried. Topical ofloxacin ointment was applied at the end of the procedure. Corneal neovascularisation developed and reached the central end of the sutures 7-10 days postoperatively. ${ }^{7}$ Three areas between two sutures were treated (fig 1). The sutures were not removed until the end of the follow up examinations. ${ }^{16}$

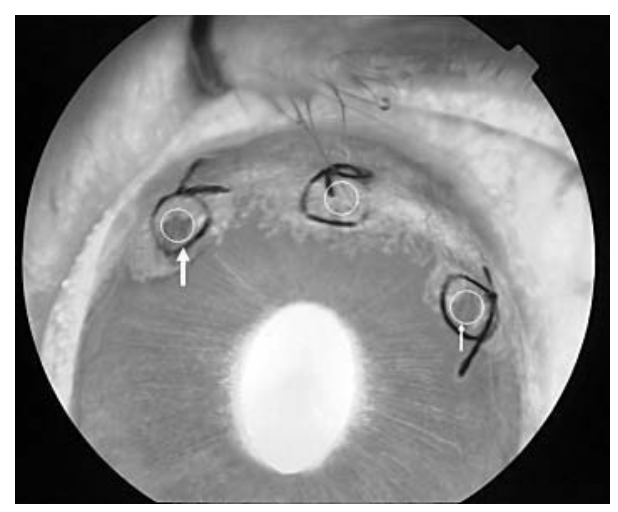

Figure 1 The fluorescein angiographic image 3 hours after PDT. The circles between two sutures are the areas that were irradiated. The left circle (large arrow) was irradiated 30 seconds after ICG injection and the right circle (small arrow) 5 minutes after ICG injection. The middle circle was untreated.

\section{ICG angiography and fluorescein angiography}

ICG angiography was performed using a fundus camera (TRC-50IX, Topcon, Tokyo, Japan), and the images were stored in a digital image processing system (IMAGEnet, Topcon). ICG was injected at a dose of $5 \mathrm{mg} / \mathrm{kg}$ with a rapid saline flush.

Fluorescein angiography was performed at a dose of $0.2 \mathrm{ml}$ of $10 \%$ sodium fluorescein solution 1 day before PDT and on days 0 ( 3 hours after treatment), 1, 3, and 10 after PDT.

\section{Photothrombosis}

Indocyanine green was reconstituted with sterile distilled water and administered at a dose of $10 \mathrm{mg} / \mathrm{kg}$ as a photosensitiser. General anaesthesia was induced in the animals as previously described. Local anaesthesia was also induced with oxybuprocaine before each experiment.

ICG was injected through the marginal ear vein. Laser irradiation was started at 30 seconds in 23 areas and 5 minutes in 22 areas after ICG injection. Because the laser instrument was not attached to the ocular probe, the laser light was guided with refracting mirrors. The irradiation parameters were as follows: peak power density, $80 \mathrm{~W} / \mathrm{cm}^{2}$ (mean power density, $38 \mathrm{~mW} / \mathrm{cm}^{2}$ ); focal spot size area, $0.047 \mathrm{~cm}^{2}$; repetition rate, $1 \mathrm{kHz}$; pulse width, 150 femtoseconds; total irradiation time, 100 seconds; and total irradiation energy, $3.8 \mathrm{~J} / \mathrm{cm}^{2}$. Before each period of irradiation, the mean power density was calibrated to $38 \mathrm{~mW} / \mathrm{cm}^{2}$.

Photodynamic therapy was usually applied to two corneal neovascularisation lesions and one corneal neovascularisation lesion was left untreated as a control (fig l). To evaluate the femtosecond laser effect alone, four eyes were irradiated at a peak power density of $80 \mathrm{~W} / \mathrm{cm}^{2}$ without ICG injection. Four eyes were left untreated to observe the natural course.

\section{Histologic examination}

We performed a histological evaluation to determine that the vessels had closed in 10 rabbits. Five rabbits at day 0, two at day 1 , and three at day 3 were sacrificed by intravenous injection of an overdose of pentobarbital sodium. The eyes were enucleated and fixed in paraformaldehyde for light microscopy and 4\% glutaraldehyde for electron microscopy for 30 minutes. The corneas and iris in the irradiated areas were then dissected from the fixed tissue. For light microscopy, the specimens were immersed again in paraformaldehyde and fixed overnight at room temperature. They were then dehydrated in ethanol, embedded in paraffin, and stained with haematoxylin eosin. For electron microscopy, the samples were immersed again and fixed overnight at $4^{\circ} \mathrm{C}$. They then were dehydrated in ethanol, fixed in osmium tetroxide, and embedded in epoxy resin. Thin sections stained with uranyl acetate and lead citrate were observed by electron microscopy (H-7000, Hitachi, Tokyo, Japan).

\section{RESULTS}

\section{Absorbance of ICG}

The optical spectrum using any ICG concentration consisted of two main peaks, mainly at $800-805 \mathrm{~nm}$ (fig 2). The absorbance value was significantly correlated with the ICG concentration at $805 \mathrm{~nm}$ (by Pearson product moment correlation, correlation coefficient $=0.979, \quad p=0.000678$ ) (fig 3).

\section{Threshold of laser power using gelatin with and without ICG}

Although gelatin without ICG did not degenerate at a peak power density of $42 \mathrm{~W} / \mathrm{cm}^{2}$ (fig $4 \mathrm{~B}$ ), it started to do so at a peak power density of $53 \mathrm{~W} / \mathrm{cm}^{2}$ (fig $4 \mathrm{C}$ ). Gelatin mixed with $1.5 \mathrm{mg} / \mathrm{dl}$ of ICG started to degenerate at a peak power 


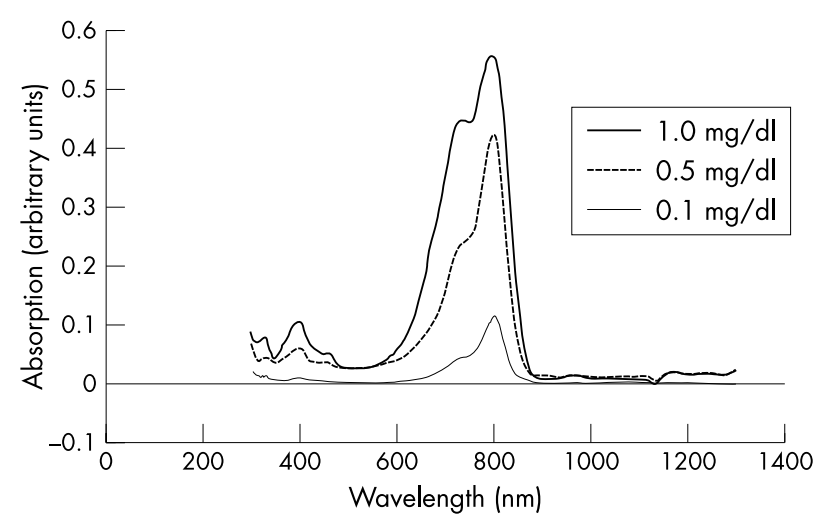

Figure 2 Absorption spectrum of ICG solution at concentrations of 0.1 , 0.5 , and $1.0 \mathrm{mg} / \mathrm{dl}$ with $0.5 \mathrm{mg} / \mathrm{dl}$ of albumin added. The main peak is at $800-805 \mathrm{~nm}$ in each concentration.

density of $39 \mathrm{~W} / \mathrm{cm}^{2}$ and degenerated at 42 and $53 \mathrm{~W} / \mathrm{cm}^{2}$ (fig 4D-F).

From both these examinations in an in vitro model, the threshold power levels at which the gelatin did not degenerate without ICG and at which it did degenerate with ICG, ranged from $39 \mathrm{~W} / \mathrm{cm}^{2}$ to $53 \mathrm{~W} / \mathrm{cm}^{2}$.

\section{ICG angiography of corneal neovascularisation}

Thirty seconds after ICG injection, ICG angiography clearly defined the network of corneal neovascularisation and iris vessels. One minute after ICG injection, the hyperfluorescence from the iris vessels decreased, and the hyperfluorescence from the corneal neovascularisation increased with enlargement of the area of leakage. Five minutes after ICG injection fluorescence from the corneal neovascularisation weakened, and disappeared from the iris.

\section{Fluorescein angiography in PDT using ICG}

The irradiation time was 30 seconds and 5 minutes after ICG injection. Twenty three lesions were irradiated after $30 \mathrm{sec}-$ onds and 22 lesions after 5 minutes.

Fluorescein angiography showed that 3 hours after PDT all 23 lesions, irradiated 30 seconds after ICG injection, were closed (fig 5B). The remaining 18 of 18 lesions were still closed at day 1 in all remaining 18 eyes (fig 5C). Irradiated lesions continued to be closed until day 10 in all remaining 12 eyes.

Of the 22 lesions, irradiated 5 minutes after ICG injection, 21 lesions were closed and only one lesion was open 3 hours

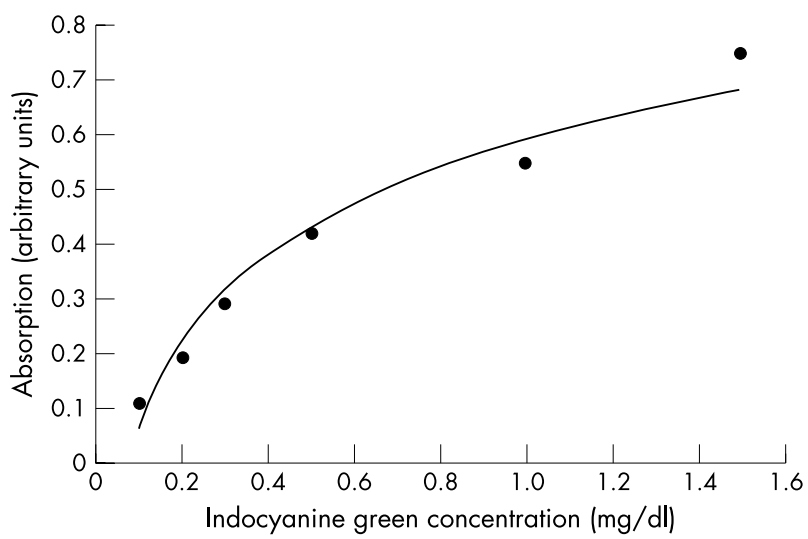

Figure 3 Absorbance value changes at $805 \mathrm{~nm}$. The absorbance values (arbitrary unit) correlate with ICG concentration. Absorption value (arbitrary unit) $=0.2301 \times(\text { ICG concentration, } \mathrm{mg} / \mathrm{dll})^{1 / 2}+0.5921$, $\mathrm{R}^{2}=0.9651$

after PDT. Fourteen of 16 lesions closed on day 3 and they closed in seven of 13 eyes on day 10. Four lesions treated using the titanium sapphire laser alone did not close in any eyes (table 1).

Corneal thinning or bubble formation was not detected by slit lamp biomicroscopy in any eyes that received laser irradiation. No photosensitivity developed in any treated animals.

\section{Histology}

Light microscopy revealed that the corneal neovascularisation was packed with erythrocytes with no invasion of inflammatory cells 3 hours after PDT (fig 6, top left). Thrombi were observed at day 3 and neovascularisation decreased (fig 6, top right). Thermal damage around the neovascularisation was not apparent. Light microscopy showed patent blood vessels without alterations in the iris 3 hours after PDT (fig 6C) and on day 3 (fig 6D).

Electron microscopy on day 3 after PDT showed cell remnants and some amorphous materials suggestive of erythrocytes in the vascular lumen (fig 7). Swelling of the endothelial cells and nuclei were detected. Nuclear alterations of the endothelial cell suggesting cell death were found. Vacuolisation of the endothelial cell cytoplasm was also observed. Neither ablation nor disorganisation of collagen fibrils was detected.
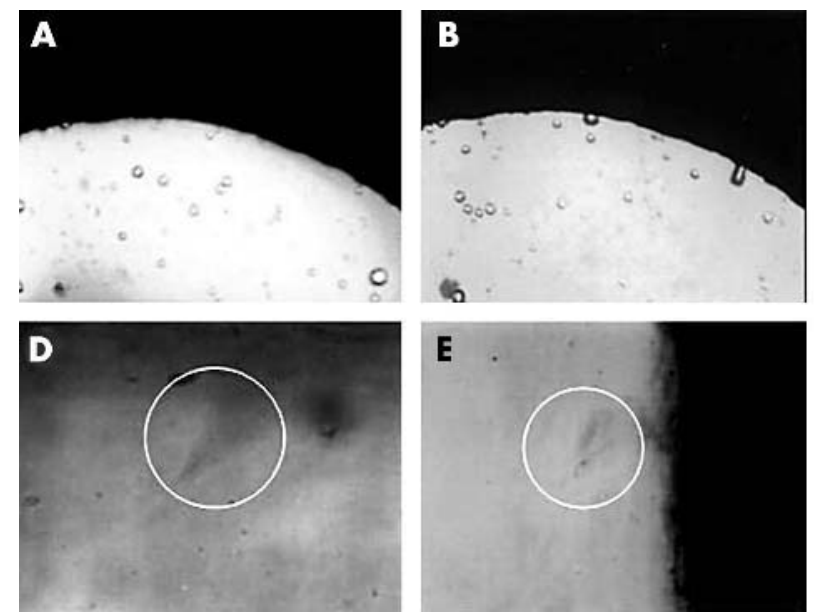

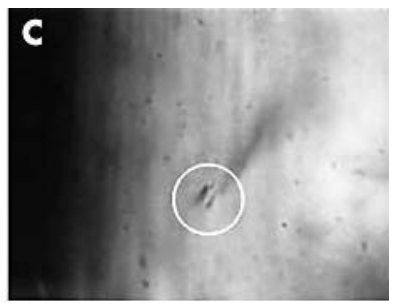

Figure 4 (A) Photograph of gelatin coagulated in cell before irradiation. (B) Gelatin remains intact at a peak power density of $42 \mathrm{~W} / \mathrm{cm}^{2}$. (C) Gelatin degeneration (circle) starts at $53 \mathrm{~W} /$ $\mathrm{cm}^{2}$. (D) Degeneration (circle) of gelatin mixed with $1.5 \mathrm{mg} / \mathrm{dl}$ of ICG starts at a peak power density of $39 \mathrm{~W} / \mathrm{cm}^{2}$.

Gelatin mixed with ICG degenerates at $42 \mathrm{~W} / \mathrm{cm}^{2}(\mathrm{E})$ and $53 \mathrm{~W} / \mathrm{cm}^{2}(\mathrm{~F})$.

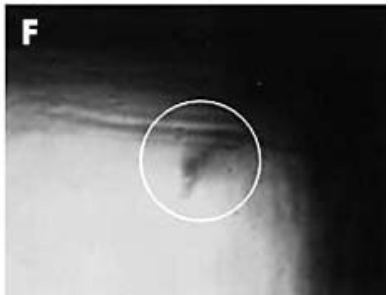




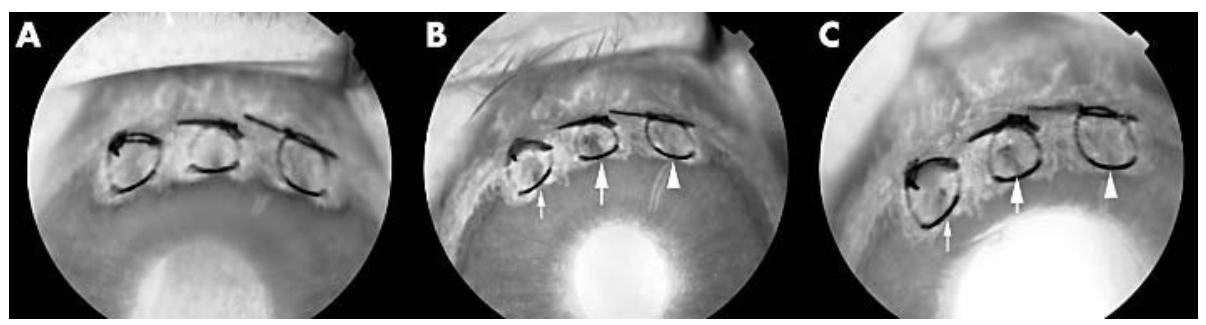

Figure 5 Fluorescein angiography 1 day before PDT $(A)$, at day 0 ( 3 hours after PDT) (B), and day 1 (C). The large arrow indicates irradiation after 30 seconds, the small arrow after 5 minutes, and the arrowhead control. The area irradiated after 30 seconds using the femtosecond pulse laser shows less hypofluorescence than the area irradiated 5 minutes after PDT.

\section{DISCUSSION}

Our results show the effects of femtosecond pulse laser with PDT enhanced by ICG using a corneal neovascularisation model. We succeeded in closing the corneal neovascularisation at $3.8 \mathrm{~J} / \mathrm{cm}^{2}$, which is very low energy. In previous reports of in vitro experiments with PDT mediated by ICG using a continuous wave laser, the authors reported using a $830 \mathrm{~nm}$ wavelength diode laser at a radiance of 30-100 J/cm $\left(100-200 \mathrm{~mW} / \mathrm{cm}^{2}\right)$ on melanoma cells ${ }^{11}$ and a $805 \mathrm{~nm}$ wavelength continuous wave diode laser at a radiance of $30 \mathrm{~J} / \mathrm{cm}^{2}\left(40 \mathrm{~mW} / \mathrm{cm}^{2}\right)$ on colon cancer cells. ${ }^{12}$

In in vivo experiments, the choriocapillaris of normal rabbits closed successfully using PDT mediated by ICG at doses of 10 and $20 \mathrm{mg} / \mathrm{kg}$ with a diode laser tuned to $810 \mathrm{~nm}$ at a radiance of $6.3 \mathrm{~J} / \mathrm{cm}^{2} .{ }^{14}$ In a clinical trial, occult choroidal neovascularisation in age related macular degeneration was successfully closed using $1.5 \mathrm{mg} / \mathrm{kg}$ of ICG at $3000 \mathrm{~mW} / \mathrm{cm}^{2}$ for 95 seconds (calculated as $285 \mathrm{~J} / \mathrm{cm}^{2}$ ). ${ }^{13}$ In Kaposi's sarcoma, 2.0 and $4.0 \mathrm{mg} / \mathrm{kg}$ of ICG were used to successfully close the lesions with a diode laser $(805 \mathrm{~nm}$ wavelength and total energy of $\left.100 \mathrm{~J} / \mathrm{cm}^{2}\right) .{ }^{17}$ Compared with previous reports, the energy level used in the present study was exceptionally low, which is the advantage of the high peak power pulse energy of the femtosecond pulse laser.

Femtosecond pulse laser has been used during refractive surgery using a photodisruption phenomenon and subthreshold laser to the retina in a few femtoseconds of irradiation. Photodisruption using the femtosecond laser is more localised than that caused by picosecond or nanosecond pulses. ${ }^{18}$ Photodisruption occurs when laser light by femtosecond pulses is focused to power intensities ranging from $10^{11}$ to $10^{12} \mathrm{~W} / \mathrm{cm}^{2}{ }^{3}$ Optical breakdown is the process by which transparent material is ionised by multiphoton absorption. ${ }^{19}$ A shock wave is created by the plasma ignition and explosive expansion. When this process occurs in a fluid, bubbles develop in a cavity. From the power range causing photodisruption, the power of this study was very low and did not generate bubble formation or ablate the tissue, making the femtosecond pulse laser much safer than other lasers.

In an in vivo study in which venous varicosities were treated by $808 \mathrm{~nm}$ continuous diode laser enhanced by ICG,

Table 1 Time dependent closure rate of corneal neovascularisation

\begin{tabular}{lllll}
$\begin{array}{l}\text { Time after } \\
\text { ICG injection } 3 \text { hours }\end{array}$ & Day $\mathbf{1}$ & Day 3 & Day 10 \\
\hline 30 seconds & $23 / 23$ & $18 / 18$ & $15 / 15$ & $12 / 12$ \\
& $(100 \%)$ & $(100 \%)$ & $(100 \%)$ & $(100 \%)$ \\
5 minutes & $21 / 22$ & $16 / 17$ & $14 / 16$ & $7 / 13$ \\
& $(95 \%)$ & $(94 \%)$ & $(88 \%)$ & $(54 \%)$ \\
Laser only & $0 / 4$ & $0 / 3$ & $0 / 3$ & $0 / 2$ \\
& $(0 \%)$ & $(0 \%)$ & $(0 \%)$ & $(0 \%)$ \\
\hline
\end{tabular}
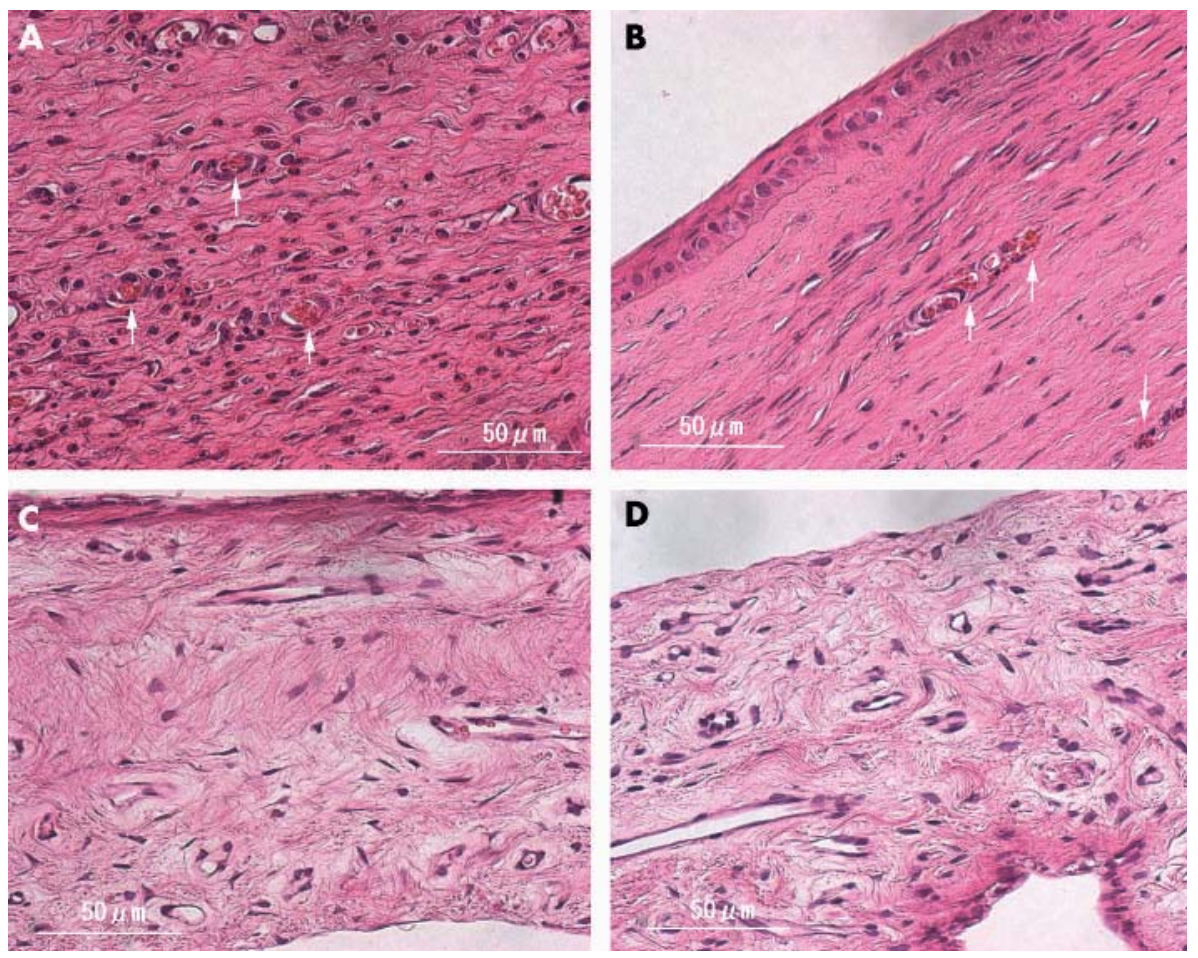

Figure 6 Light micrograph of a cornea (top) and an iris (bottom) stained with haematoxylin eosin at day 0 (left) and day 3 (right). (A) Corneal neovascularisation is filled with thrombus (arrow) with no severe inflammatory changes. (B) Small vessels are packed with aggregated erythrocytes and platelets (arrow). No inflammatory changes are detected and there is a small cavity of

neovascularisation. Iris vessels are patent and no morphologic changes are seen at day $0(C)$ or day $3(D)$ (haematoxylin eosin staining, original magnification $\times 400$ ). 


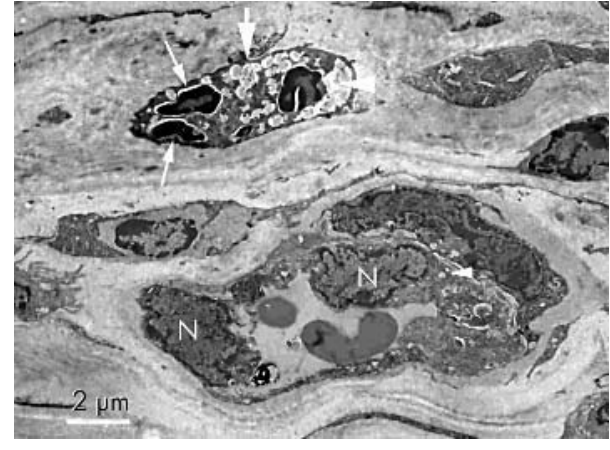

Figure 7 Transmission electron micrograph of cornea on day 3 after photodynamic therapy. Cell remnants and some amorphous materials (large arrow) are packed in the vascular lumen (large arrow head). Nuclear alterations of endothelial cells (small arrow) are seen.

Vacuolisation of the endothelial cell cytoplasm (small arrow head) and swelling of endothelial nuclei (N) are also observed (uranyl acetate and lead citrate $\times 2000$ ).

the histological results of light microscopy showed an area of focal thermal damage surrounding the vessels..$^{20}$ Temperature increases ranging from $5-25^{\circ} \mathrm{C}$ within tumours were reported after PDT using haematoporphyrin derivative, and a thermal effect on normal tissue was suggested. ${ }^{21}$ The ideal effect of PDT is no or minimal damage to the surrounding normal tissue from the secondary thermal effect. Our histological results did not show thermal damage in surrounding tissue.

From a report that analysed time resolved fluorescence spectroscopy using ICG, the response time from irradiation to the maximum signal fluorescence was approximately l nanosecond, and no fluorescence signal was detected at approximately 4 nanoseconds. ${ }^{22}$ The interval between each laser peak in the present study was 1 millisecond calculated from the repetition rate of $1 \mathrm{kHz}$, which might be sufficient to cool down surrounding normal tissue to prevent thermal spread. Continuous wave laser has no cooling effect. Considering this, femtosecond pulse laser might be the ideal laser for PDT. Interruptions in the femtosecond pulse laser really help to reduce the thermal effect without decreasing the photodynamic effect, compared with the continuous wave laser.

The disappearance rates of ICG in healthy subjects ranged from $18 \%$ to $24 \%$ per minute and the normal biological half life is approximately 3 minutes. The ICG concentration at 5 minutes was estimated to be approximately $30 \%$ of the initial ICG concentration. At day 10, occlusion of new vessels was seen in $100 \%(12 / 12)$ when tissue was exposed to the laser for 30 seconds after ICG injection, and the closure rate was $54 \%(7 / 13)$ when the laser was applied 5 minutes after ICG injection. It is reasonable that laser exposure is more effective at 30 seconds than 5 minutes after ICG injection when one considers ICG metabolism and the correlation between ICG absorption and concentration (fig 3).

To obtain the optimal goal of closing only the neovascular lesions and not injuring the surrounding normal tissue, various parameters must be examined-for example, the photosensitiser dose, the timing of the start of irradiation, laser power, and laser duration. In preliminary experiments, we performed PDT using ICG at a dose of $1.0 \mathrm{mg} / \mathrm{kg}$ and at a peak power density of $53 \mathrm{~W} / \mathrm{cm}^{2}$. With these parameters, no occlusion was obtained in any eyes (unpublished data). We used ICG at a dose of $10 \mathrm{mg} / \mathrm{kg}$, the concentration reported by Costa et al. ${ }^{14}$ An intravenous LD50 of ICG in a rat was reported to range from $50-80 \mathrm{mg} / \mathrm{kg}^{23}$ concentrations that are much higher than that used in our study. Determining the optimal concentration of ICG should be addressed before a clinical trial is performed, although less ICG might induce less thermic reaction following less of a photodynamic reaction.

We chose the time to start the first irradiation at 30 seconds following the results of ICG angiography of corneal neovascularisation. We set the second irradiation at 5 minutes because of the total irradiation time of $100 \mathrm{sec}-$ onds. For example, the irradiation time starting at 1 minute overlaps 70 seconds with the time starting at 30 seconds. In this study, we used a laser power in the in vivo experiments that was determined from the results of threshold experiments performed in vitro using gelatin mixed with $1.5 \mathrm{mg} / \mathrm{dl}$ of ICG. Injected ICG was diluted within the rabbit body and the highest concentration soon after the injection was estimated to be approximately $1 \mathrm{mg} / \mathrm{dl}$ of ICG. Based on the correlation between absorption and ICG concentration (fig 3), the peak power was set at $80 \mathrm{~W} / \mathrm{cm}^{2}$, which is $53 \mathrm{~W} / \mathrm{cm}^{2}$ multiplied by 1.5 , from the difference of the ICG concentrations between the in vitro and the in vivo experiments.

Further investigations are required to confirm that PDT using the femtosecond pulse laser and ICG can be used clinically to treat neovascular tissues.$^{24}$ Further, this study did not prove long term efficacy, as the follow up time was only 10 days. Longer follow up is needed to show advanced efficacy.

The femtosecond pulse laser might be a potential treatment modality used for PDT with ICG and it may herald an era of new treatments.

\section{ACKNOWLEDGEMENTS}

The authors thank Yuji Nishida and Masashi Teranishi, Institute of Free Electron Laser, Graduate School of Engineering, Osaka University, for their in vitro experimental work; Kaho Yoshida, the Department of Ophthalmology, Osaka University Medical School, for histological work, and Eiji Oiki, technical staff of the Center for Research and Education, Osaka University Graduate School of Medicine, for electron microscopic work.

\section{Authors' affiliations}

M Sawa, T Takahashi, H Sakaguchi, M Ohii, Y Tano, Department of Ophthalmology and Visual Science, Osaka University Graduate School of Medicine, Osaka, Japan

K Awazu, H Horiike, Institute of Free Electron Laser, Graduate School of Engineering, Osaka University, Osaka, Japan

Supported by grant No. 15591855 from the Ministry of Education and, Science and Culture of Japan (MO).

\section{REFERENCES}

1 Diels J, Rudolph W. Ultrashort laser pulse phenomena: Fundamentals, techniques, and applications on a femtosecond time scale. San Diego, California: Academic Press, 1996.

2 Thomas RJ, Noojin GD, Stolarski DJ, et al. A comparative study of retina effects from continuous wave and femtosecond mode-locked lasers. Lasers Surg Med 2002;31:9-17.

3 Lubatschowski H, Maatz G, Heisterkamp A, et al. Application of ultrashort laser pulses for intrastromal refractive surgery. Graefes Arch Clin Exp Ophthalmol 2000;238:33-9.

4 Tirlapur UK, Konig K. Targeted transfection by femtosecond laser. Nature 2002;418:290-1.

5 Zeira E, Manevitch A, Khatchatouriants A, et al. Femtosecond infrared laseran efficient and safe in vivo gene delivery system for prolonged expression. Mol Ther 2003;8:342-50.

6 Schmidt-Erfurth U, Laqua H, Schlotzer-Schrehard U, et al. Histopathological changes following photodynamic therapy in human eyes. Arch Ophthalmol 2002; 120:835-44.

7 Schmidt-Erfurth U, Hasan T, Schomacker K, et al. In vivo uptake of liposomal benzoporphyrin derivative and photothrombosis in experimental corneal neovascularization. Lasers Surg Med 1995;17:178-88.

8 Photodynamic therapy of subfoveal choroidal neovascularization in agerelated macular degeneration with verteporfin: one-year results of 2 randomized clinical trials -TAP report. Treatment of age-related macular degeneration with photodynamic therapy (TAP) Study Group. Arch Ophthalmol 1999;117:1329-45.

9 Benya R, Quintana J, Brundage B. Adverse reactions to indocyanine green: a case report and a review of the literature. Cathet Cardiovasc Diagn 1989; 17:231-3. 
10 Slakter JS, Yannuzzi LA, Guyer DR, et al. Indocyanine-green angiography. Curr Opin Ophthalmol 1995;6:25-32.

11 Urbanska K, Romanowska-Dixon B, Matuszak Z, et al. Indocyanine green as a prospective sensitizer for photodynamic therapy of melanomas. Acta Biochim Pol 2002:49:387-91.

12 Baumler W, Abels C, Karrer S, et al. Photo-oxidative killing of human colonic cancer cells using indocyanine green and infrared light. Br J Cancer 1999;80:360-3.

13 Costa RA, Farah ME, Cardillo JA, et al. Photodynamic therapy with indocyanine green for occult subfoveal choroidal neovascularization caused by age-related macular degeneration. Curr Eye Res 2001;23:271-5.

14 Costa RA, Farah ME, Freymuller E, et al. Choriocapillaris photodynamic therapy using indocyanine green. Am J Ophthalmol 2001;132:557-65.

15 Tsilimbaris MK, Pallikaris IG, Naoumidi II, et al. Phthalocyanine mediated photodynamic thrombosis of experimental corneal neovascularization: effect of phthalocyanine dose and irradiation onset time on vascular occlusion rate. Lasers Surg Med 1994;15:19-31.

16 Gohto Y, Obana A, Kanai M, et al. Photodynamic therapy for corneal neovascularization using topically administered ATX-S10 (Na). Ophthalmic Surg Lasers 2000;31:55-60.
17 Abels C, Karrer S, Baumler W, et al. Indocyanine green and laser light for the treatment of AIDS-associated cutaneous Kaposi's sarcoma. Br J Cancer 1998;77:1021-4

18 Juhasz T, Kastis GA, Suarez C, et al. Time-resolved observations of shock waves and cavitation bubbles generated by femtosecond laser pulses in corneal tissue and water. Lasers Surg Med 1996;19:23-31.

19 Vogel A. Nonlinear absorption: intraocular microsurgery and laser lithotripsy. Phys Med Biol 1997; 42:895-912.

20 Libutti SK, Oz MC, Chuck RS, et al. A preliminary study of dye-enhanced laser photosclerosis. Surgery 1991;109:163-8.

21 Gomer CJ, Ferrario A, Hayashi N, et al. Molecular, cellular, and tissue responses following photodynamic therapy. Lasers Surg Med 1988;8:450-63.

22 Sadoqi M, Riseborough P, Kumar S. Analytical models for time resolved fluorescence spectroscopy in tissues. Phys Med Biol 2001;46:2725-43.

23 Lutty GA. The acute intravenous toxicity of biological stains, dyes, and other fluorescent substances. Toxicol Appl Pharmacol 1978;44:225-49.

24 Pallikaris IG, Tslimbaris MK, lliaki OE, et al. Effectiveness of corneal neovascularization photothrombosis using phthalocyanine and a diode laser (675 nm). Lasers Surg Med 1993;13:197-203. 